\title{
Factors Associated with Consumer Knowledge of Garlic Health Properties, Garlic Preparation Knowledge and Garlic Intake
}

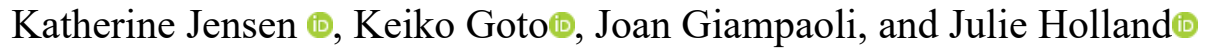 \\ Dept. of Nutrition and Food Sciences California State University, Chico
}

\begin{abstract}
Introduction and Background: Garlic has been shown to provide numerous health benefits attributed to its innate compound allicin. However, this compound is highly unstable when exposed to various cooking methods as it is affected by temperature and $\mathrm{pH}$ changes. There is limited information about health and preparation knowledge of garlic among consumers with various nutrition education levels including future nutrition professionals such as registered dietitian nutritionists. This study determined if consumer garlic health knowledge and perceptions were associated with garlic intake and if nutrition education level was associated with garlic health and preparation knowledge. Methods: An online survey was conducted to examine garlic health and preparation knowledge, garlic health perceptions, garlic intake, and garlic cooking methods among undergraduate students with and without a major. Results: There was a significant moderate positive correlation between garlic health knowledge and garlic intake $\left(\mathrm{r}_{\mathrm{s}}=.421, \mathrm{p}<.001\right)$. Those with a nutrition major had significantly greater garlic health knowledge than non-majors $(\mathrm{p}<0.001)$, while there was no difference between nutrition majors and non-majors on cooking methodology knowledge. Conclusion: Knowledge of the health benefits of garlic appears to be associated with its intake. Nutrition education should focus more on appropriate garlic preparation in relation to its health benefits.
\end{abstract}

(C) 2021 and CC-BY 4.0 licensed by the authors.

Keywords: Garlic, Allicin, Cooking Methods, Nutrition Knowledge

\section{Introduction}

Functional foods are whole, fortified, enriched or enhanced foods widely used in the diet that when consumed regularly are said to have long term beneficial health effects such as the reduction in the risk of diabetes, cardiovascular disease (CVD) and cancer Examples of functional foods include whole foods like oats, garlic and green teas as well as enriched or fortified products like probiotic yogurt or fortified cereals (Lobo, Patil, Phatak \& Chandra, 2010; Di Pasquale, Adinolfi \& Capitanio, 2011). Functional foods contain biomolecules such as phytochemicals, nonstarchy carbohydrates, and probiotics and prebiotics which act to positively alter metabolic processes resulting in reported beneficial health effects. Consumers are aware of many functional food benefits. One study investigating consumer perceptions of functional foods found that the incentives to purchase functional foods were primarily based in the belief that they could increase overall health and longevity, as well as reduce the risk of various medical conditions (Teoh, Ngorsuraches, Lai, Bangpan \& Chaiyakunapruk, 2019). However, the consumption functional foods do not necessarily coincide with an increased acquisition of health benefits, as cooking and preparation techniques can both positively and negatively alter the metabolic activity of the functional components (Lee, Choi, Jeong, Lee, \& Sung, 2017). 
Garlic (Allium sativum L.) is a popular functional among diverse cultures (Lee et al., 2017; AstonMorgan \& Wickett, 2018). It is a bulb-forming vegetable belonging to the Alliacea family (e.g., onions, leeks, shallots) that has had an integral role in various medicinal practices across various cultures and time, dating back as early as 2700 B.C. (Locatelli, Altamirano, González \& Camargo, 2015; Mnayer, Fabiano-Tixier, Petitcolas, Hamieh, Nehme, et al., 2014). In modern times, numerous epidemiological studies have shown garlic to effectively produce antimicrobial and antioxidant effects as well as provide therapeutic and preventative measures in the treatment of cancer, diabetes and most notably CVD (Petrovska \& Cekovska, 2010). Garlic, like many functional foods and their respective functional components, contains a compound that is primarily responsible for the reported health benefits known as allicin (Bayan, Koulivand \& Gorji, 2014).

Allicin (diallyl thiosulfinate) is a reactive organosulfur compound present in whole garlic bulbs in its fixed precursor form, alliin (Rahman, 2007). When garlic tissue is damaged through processes including chewing or cutting, the alliinase enzyme is released which acts to convert alliin into allicin (Rahman, 2007). Allicin is credited for the numerous beneficial health properties of garlic including its ability to increase cellular antioxidant capacity, produce anti-carcinogenic effects, and reduce CVD risk (Chan, Yuen, Chan, \& Chan, 2013). Though allicin has been shown to have numerous health benefits, many harvesting and distribution elements of garlic production from producer cultivation practices to consumer cooking methods can greatly alter garlic allicin content.

One of the most efficient ways to consume garlic to receive therapeutic amounts of allicin is through the ingestion of freshly minced/crushed cloves. However, preparations with lower allicin content (e.g., pre-minced and powdered forms) have been shown to be utilized much more frequently (Aston-Morgan et al., 2018; Rahman, 2007; Martins et al., 2016). Fresh garlic can contain anywhere from 4.30 to $8.99 \mathrm{mg} / \mathrm{g}$ while other forms like garlic powder show lower levels as a result of alliinase enzyme degradation during dehydration (Silva, Moretti \& Mattos, 2010; Rahman, 2007). Allicin is a highly unstable compound that partially degrades in specific cooking mediums, with temperature and $\mathrm{pH}$ changes, and when cooked for extended periods of time (Locatelli et al., 2015; Chan et al., 2013). Degradation of this compound is prevalent when stir-fried and when boiled and steamed with stirfrying/sautéing methods being the most damaging, followed by boiling and then steaming (Locatelli et al., 2015).

Aston-Morgan and Wickett, (2018) investigated consumers' understanding of herbs and spices as functional foods and found garlic to be among the top three herbs/spices reported by consumers to provide health benefits (Aston-Morgan et al., 2018). Consumers' specific understanding of garlic health benefits is limited depending on the population. One study found that over $90 \%$ of consumers were unaware or unsure of garlic's potential to reduce CVD risk (Aston-Morgan et al., 2018), while another study found that $20 \%$ of participants were aware of the garlic-CVD relationship (Isbill, Kandiah \& Khubchandani, 2017). Another study reported that roughly half of the study participants consumed garlic on a regular basis and believed it to provide health benefits (Aston-Morgan et al., 2018). However, there is no known study that has assessed consumers' understanding of the nutritionally beneficial preparation methods of functional foods such as garlic. This is likely because functional food is a relatively new concept in the field of nutrition. It is necessary for nutrition professionals to be aware of proper methods for preparing functional food so that they can help consumers optimize its health benefits.

\section{The Current Study}

Increasing garlic intake and using proper preparation methods that retain allicin content in garlic are two important ways of maximizing health benefits of garlic. This study investigated 
consumers' garlic health and preparation knowledge as a focal point. Consumers may be knowledgeable of garlic's health benefits and ingest garlic for health purposes but may be unaware of preparation methods that affect allicin content. Consumers investigated in this study included both undergraduate students with high nutrition education level (nutrition majors) and students with low nutrition education level (nonnutrition majors) from a public university. This study also aimed to determine if nutrition education through undergraduate nutrition courses play a significant role in obtaining both scientific health and preparation knowledge of garlic among participants.

\section{Methods}

\section{Study Design}

This was a cross-sectional study assessing undergraduate students' knowledge and intake of garlic. An online survey was administered via Qualtrics. The use of human subjects for this study was approved by the Institutional Review Board (IRB) at [University].

\section{Participants}

Study participants included a convenience sample of undergraduate students from two nutrition courses in spring 2020, in a large public university. One course was an introductory nutrition course for non-nutrition students of all class standings, while the other course was a senior level nutrition course consisting of mainly upper level nutrition majors Non-nutrition major participants in an introductory nutrition course were selected to better ensure a homogenous sample of participants with similar levels of nutrition education and interests in nutrition. A total of 250 participants in these courses were sent the survey link for this research through their respective instructors. Seventy-one students $(28 \%)$ completed the survey.

\section{Measures and Procedures}

Overview of Survey Instrument. The survey utilized for this study was comprised of eight different sections that assessed demographics, garlic health knowledge, garlic preparation knowledge, garlic intake, interest in healthy eating, nutrition education, perceptions of garlic health, and primary garlic cooking/preparation methodology. The demographics section assessed gender, age, ethnicity, major (nutrition major or non-nutrition major) and year in college. There were 10 questions that assessed participants' garlic health knowledge with statements such as "garlic has been shown to help reduce the risk of developing cardiovascular disease" with response options that consisted of True or False. The number of correctly answered questions were added up, for a possible total score ranging from $0-10$. These questions were derived from a study that investigated consumers' general nutrition knowledge (Dickson-Spillmann, Siegrist \& Keller, 2011).

Garlic Preparation Knowledge. There were 8 questions that assessed participants' garlic preparation knowledge with statements including "cooking garlic is a better way to get health benefits compared to eating fresh garlic" with "True" and "False" response options. Theses knowledge questions were derived from a study that investigated consumer knowledge of dietary supplements (Karbownik, Paul, Nowicka, Nowicka, Kowalczyk, et al., 2019). The number of correctly answered questions were added up, for a possible total score ranging from 0-8.

Garlic Intake. Frequency of general garlic intake which asked participants how often they eat garlic. Answers were: "a few times a day", "daily", "a few times a week", "weekly", "a few times a month", "monthly", and "rarely/never."

Interest in Healthy Eating. There were four questions that assessed participants' interest in healthy eating, with statements that include, "It is important to me that the foods I eat daily are nutritious" with response options that ranged from "strongly disagree" to "strongly agree." This section was derived from a previously conducted study that assessed fish consumption (Pieniak, Verbeke, \& Scholderer, 2010). Responses were categorized into 2 groups with 0 - 
2 responses on average as "low or no interest in healthy eating" and 3-4 responses showing interest in healthy eating as "medium or high interest in healthy eating."

Garlic Health Perception. Participants were prompted to select yes or no on a garlic health perception question that asked if they believed garlic to be a healthy food, a question also derived from the study assessing fish consumption (Pieniak et al., 2010). Belief that garlic is a healthy food was split into two categories of "belief" and "no belief" based on participants responses of yes or no.

Garlic Preparation Methods. Participants were asked to select their primary garlic preparation/cooking method from responses that included fresh, heated in oil/fat, steamed, roasted, powdered (garlic powder, not garlic salt), preminced (in jar) and supplement. These garlic preparation types were chosen based on a previous assessment of consumer garlic usage as well as a study that investigated allicin content in various forms of garlic preparations (Locatelli et al., 2015; Ashraf et al., 2011). Primary garlic cooking methodology responses were grouped into three separate categories based on previously reported allicin content levels (Aston-Morgan et al., 2018; Locatelli et al., 2015; Rahman, 2007; Martins et al., 2016). Category 1 was comprised of garlic preparation types that have been previously reported as having higher Allicin content compared to other options which included "fresh," "pre-minced" and "supplement" while Category 2 had a relative medium amount of allicin which included "roasted," "steamed" and "baked". Category 3 was made of "heated in fat/oil" which has been reported to have the lowest Allicin content out of all the garlic preparation choices.

The link to the online survey was sent out to participants in the two nutrition courses through campus email by their respective course lecturers and professors. A total of 250 participants received the email link and were given two weeks to complete the survey. Eighty-two students participated in the survey, but responses from eleven participants were excluded as they only completed the demographics portion of the survey. Due to this exclusion, the total sample size for data analysis was seventy-one participants.

\section{Data Analysis}

Data analysis was performed using IBM SPSS Statistics Version 25. Spearman's correlation was used to determine the correlation between consumer garlic health or preparation knowledge levels as associated with and garlic intake. Ttests were used to compare gender difference on garlic health knowledge and garlic preparation knowledge. Multinomial logistic regression was performed to determine the association between garlic preparation knowledge level and primary garlic cooking methodology. The Mann-Whitney test was used to determine gender differences on garlic intake, as well as to determine consumer interest difference on garlic intake level. Ethnicity was recoded into three categories that included White, Hispanic and Other. ANOVA was used to assess ethnic differences on garlic health knowledge. The independent t-test was used to determine if consumer interest in healthy eating was associated with their garlic health knowledge level. Finally, t-tests were used to determine if nutrition education level (nutrition major versus non-nutrition major) determined both garlic health knowledge and garlic preparation knowledge.

\section{Results}

\section{Demographic Characteristics}

A total of 71 students participated in the study and the demographics of the study participants are shown in Table 1. Roughly $78.9 \%$ of the participants were female and $85.9 \%$ were aged between 18 and 24 years old. Most participants listed themselves as being either Freshmen or Seniors, and $36.6 \%$ of participants reported themselves as nutrition majors. A majority of the participants identified as either White $(56.3 \%)$ or Hispanic/Latino (32.4\%). 
Table 1.

Demographics of Participants $(n=71)$

\begin{tabular}{lc}
\hline Characteristics & Percent (n) \\
\hline Gender & $78.9(56)$ \\
Female & $21.1(15)$ \\
Male & \\
Age & $85.9(61)$ \\
$18-24$ & $11.3(8)$ \\
$25-34$ & $2.8(2)$ \\
$35-44$ & \\
Ethnicity/Race & $56.3(40)$ \\
White & $32.4(23)$ \\
Hispanic or Latino or Spanish Origin & $1.4(1)$ \\
Black or African American & $1.4(1)$ \\
Native American or Alaskan Native & $8.5(6)$ \\
Asian & $0(0)$ \\
Native Hawaiian or Pacific Islander & \\
Year in School & $33.8(24)$ \\
Freshmen & $12.7(9)$ \\
Sophomore & $8.5(6)$ \\
Junior & $39.4(28)$ \\
Senior & $5.6(4)$ \\
Graduate & \\
Nutrition Major & $36.6(26)$ \\
Yes & $63.4(45)$ \\
No &
\end{tabular}

\section{Garlic Health Knowledge and Intake}

The mean score of participant garlic health knowledge was 6.31 (S.D. \pm 1.60 ) with a range of scores from 1-10. The most frequent garlic intake response with $36.6 \%$ of responses was "few times a week" followed by "daily" with $18.3 \%$ and then "few times a month" with $15.5 \%$. A significant moderate positive correlation between garlic health knowledge levels and garlic intake levels was found $\left(\mathrm{r}_{\mathrm{s}}=.421, \mathrm{n}=71, \mathrm{p}<.001\right)$. There were no gender differences on garlic health knowledge $(\mathrm{p}=.634)$ and neither was ethnicity $(\mathrm{p}=0.09)$. There were no gender differences on garlic intake $(p=0.25)$; nor were there any ethnic differences $(\mathrm{p}=0.25)$.

The Mann-Whitney $U$ test revealed that there were no significant associations between consumer interest in healthy eating and garlic intake levels $(U=303.00, \quad p=0.658)$. The association between garlic health perceptions and garlic intake levels was also assessed. Only five participants indicated that they did not believe garlic to be a health food. Interestingly, the Mann-Whitney U test revealed that garlic intake was lower for participants who believed garlic to be a healthy food compared to those who did not believe garlic to be a healthy food ( $U=55.5$, $\mathrm{p}=0.011$ ).

\section{Garlic Preparation Knowledge}

The mean score of participant garlic preparation knowledge was 5.88 (S.D. \pm 1.42 ) with a range of scores from 3-8. Women were found to have significantly higher garlic preparation knowledge $(\mathrm{M}=6.08, \mathrm{SD}=1.43)$ compared to men $(\mathrm{M}=5.14$, $\mathrm{SD}=1.17)(\mathrm{p}=0.028)$. The most common group 
of primary garlic cooking method was category 2 (steamed, roasted and powdered) with $45.1 \%$ of respondents selecting this category. A multinomial logistic regression was performed to model the relationship between garlic preparation knowledge and the three groups of primary garlic preparation methods. Garlic preparation knowledge was not significantly associated with garlic preparation methods $(\mathrm{p}=.617)$.

\section{Associations between Nutrition Education Levels and Garlic Knowledge}

Table 2 shows associations between nutrition education levels and garlic health knowledge and garlic preparation knowledge levels. The independent t-tests revealed that garlic health knowledge was found to be significantly higher in nutrition majors compared to non-nutrition majors $(p<0.001)$. Those in the high nutrition education group were found to have higher levels of garlic health knowledge $(\mathrm{M}=7.23, \mathrm{SD}=1.48)$ compared to those in the low nutrition education group $(\mathrm{M}=5.78, \mathrm{SD}=1.43)$ and this difference was significant $(\mathrm{p}<0.001)$. Those in the high nutrition education group $(\mathrm{M}=6.12, \mathrm{SD}=1.51)$ were found to have higher levels of garlic preparation knowledge compared to those in the low nutrition education group $(\mathrm{M}=5.73$, $\mathrm{SD}=1.36)$ but this difference was not significant $(p=0.28)$. Finally, the Mann-Whitney $U$ test revealed that garlic intake was significantly higher in the high nutrition education group compared to the low nutrition education group $(\mathrm{U}=352, \mathrm{p}=0.004)$.

Table 2: Association between Garlic Knowledge and Nutrition Education Level

\begin{tabular}{lllll}
\hline Garlic Health Knowledge* & $\mathrm{n}$ & Mean & SD & $\mathrm{p}$ \\
\hline High Nutrition Education & 26 & 7.23 & 1.48 & $<0.001$ \\
Low Nutrition Education & 45 & 5.78 & 1.43 & \\
\hline $\begin{array}{l}\text { Garlic Preparation } \\
\text { Knowledge** }\end{array}$ & & & & 0.284 \\
\hline High Nutrition Education & 26 & 6.12 & 1.51 & \\
Low Nutrition Education & 41 & 5.73 & 1.36 & \\
\hline $\begin{array}{l}* \text { Garlic health knowledge graded on scale from 0-10 (0 being no knowledge and } 10 \text { being high knowledge }) \\
\text { ** Garlic preparation knowledge graded on scale from 0-8 (0 being no knowledge and } 8 \text { being high knowledge })\end{array}$
\end{tabular}

\section{Discussion}

The objective of this study was to examine factors associated with consumers' garlic health knowledge, garlic preparation knowledge and garlic intake. Other factors associated with garlic consumption and preparation such as nutrition education level, consumer interest in healthy eating and belief that garlic is a healthy food were also investigated relative to garlic health and preparation knowledge. To our understanding, this is one of the first studies that have investigated knowledge and intake of functional foods among college students including nutrition majors.

There was a significant moderate positive correlation between objective measures of garlic health knowledge level and garlic intake. Previous studies have produced mixed results for health food intake in relation to health knowledge, with stronger relationships found for subjective or perceived knowledge of nutrition and health (Pieniak, et al., 2010). Unlike previous studies (e.g., Spronk, 2014), we found no significant ethnic or gender differences in 
relation to garlic health knowledge and intake. Overall, the positive association with garlic health knowledge and garlic intake shown in this study indicates the importance of consumer health knowledge in influencing the consumption of functional foods.

The current study revealed that women had significantly higher garlic preparation knowledge levels compared to men. Past research has shown that higher levels of cooking skills are positively associated with healthier diets (Hartmann, Dohle $\&$ Siegrist, 2013; Ternier, 2010). Women tend to cook for their families significantly more than men(García-González, Achón, Alonso-Aperte, \& Varela-Moreiras, 2018). There was no significant relationship between garlic preparation knowledge and primary preparation methods. This finding indicates that there may be other factors that influence garlic preparation methods, such as socio-economic status or cultural and culinary practices, that were not examined in this study.

Unlike previous studies, we found no significant association between consumer interest in healthy eating and garlic intake level. One study found that participant interest in healthy eating had a weak positive relationship with fish consumption (Pieniak et al., 2010). Though previous studies have reported positive associations between interest in healthy eating or healthy lifestyles and healthy dietary intake (e.g., perceived motivation towards health and calcium intakes among postmenopausal women, Edmonds, Turner, \& Usdan, 2012), the lack of relationship with garlic might be due to various other factors. Garlic might be consumed for other reasons besides health, such as for taste as one study found that participants ranked factors such as taste, color and smell over health qualities when purchasing foods (Schroeter, House \& Lorence, 2007).

Those in the high nutrition education group had significantly higher levels of both garlic intake and garlic health knowledge compared to those in the low nutrition education group, similar to the findings of Nani (2016). Another study found that nutrition majors had significantly higher scores on nutritional knowledge assessments compared to physical science majors (Choi \& Rha, 2008).

Participants in the high nutrition education group were found to have higher levels of garlic preparation knowledge compared to those in the low nutrition education group, but this difference was not significant. Short (2003) discussed that one's ability to cook is comprised of many various facets of cooking outside of the basic mechanical skills required to cook a meal (Short, 2003). These facets include technical, perceptual, conceptual, organizational, and academic knowledge (Short, 2003). This current assessment indicates that preparation knowledge could be a major barrier in the ability of consumers to properly prepare their foods in ways to receive the most health benefits. This barrier even extends to nutrition majors, who are are educated on health properties of functional foods such as garlic, but are not taught how to prepare such foods in an optimal way. Dietetic professionals should be aware of the effects of preparation/cooking on food so that they can best inform their clients on how to optimize health benefits of functional foods.

\section{Limitations}

The utilization of an online survey is a limitation with this study as it produced a low response rate. The small sample size with a low response rate might also have produced data that is not fully representative of the sample population or created lack of relationships that might have been seen in a larger sample size. Income was not assessed and could potentially be a factor in consistently consuming garlic. Lastly, this study only assessed students from one university and the study results of the current study cannot be extrapolated to students in other universities or consumers in different age groups.

\section{Conclusions}

While young consumers might understand the health properties and benefits of various functional foods, they might not know how the preparation methods of these foods can greatly 
affect health benefits. The current study did find a significant positive correlation between garlic health knowledge and garlic intake, which indicates the importance of nutrition education among consumers. However, consumer knowledge about the health benefits and their respective increased intake of functional food might not automatically lead to receiving its optimal health benefits if consumers are preparing foods in ways that negatively affect beneficial constituents. Further research needs be conducted to assess health and preparation knowledge of other functional foods besides garlic.

Based on results of the current study, it is possible that this knowledge is lacking in nutrition curriculum. It is important that consumers interested in healthy eating be informed of proper preparation methods so that they may receive the full health benefits of functional foods. More research is needed to examine health and preparation knowledge of functional foods among nutrition majors and dietetic professionals such as registered dietitians.

\section{References}

Ashraf, Rizwan \& Khan, Rafeeq \& Ashraf, Imran. (2011). Garlic (Allium sativum) supplementation with standard antidiabetic agent provides better diabetic control in type 2 diabetes patients. Pakistan Journal of Pharmaceutical Sciences, 24(4), 565-70. website, https://pubmed.ncbi.nlm.nih.gov/21959822/

Aston-Morgan, M., \& Wickett, H. (2018). Consumer perceptions and intakes of garlic as a cardio-protective food. Centre of Nutrition and Dietetics, Cardiff, Metropolitan University Cardiff. doi: 10.1016/j.appet.2015.11.001.

Bayan, L., Koulivand, P. H., \& Gorji, A. (2014). Garlic: a review of potential therapeutic effects. Avicenna Journal of Phytomedicine, 4(1), 1-14.website, https://pubmed.ncbi.nlm.nih.gov/25050296/

Chan, J., Yuen, A., Chan, R., \& Chan, SW. (2013). A Review of the cardiovascular benefits and antioxidant properties of allicin. Phytotherapy Research, 27(5), 637-46. doi:10.1002/ptr.4796.

Chang S. F. (2006). A cross-sectional survey of calcium intake in relation to knowledge of osteoporosis and beliefs in young adult women. International Journal of Nursing Practice, 12(1), 21-27. doi: 10.1111/j.1440-172X.2006.00546.x

Choi, J. M. \& Rha H. B. (2008). A study on the nutrition knowledge and nutritional status of food and nutrition major and physical science major female students. Korean Journal of Community Nutrition, 13(1), 24-33. doi: 10.4162/nrp.2008.2.4.308

Di Pasquale, J., Adinolfi, F., \& Capitanio, F. (2011). Analysis of consumer attitudes and consumers' willingness to pay for functional foods. International Journal on Food System Dynamics, 2(2). doi:10.18461/ijfsd.v2i2.227.

Dickson-Spillmann, M., Siegrist, M., \& Keller, C. (2011). Development and validation of a short, consumer-oriented nutrition knowledge questionnaire. Appetite, 56(3), 617-20. doi: 10.1016/j.appet.2011.01.034.

Edmonds, E., Turner, L., \& Usdan, S. (2012). Osteoporosis knowledge, beliefs, and calcium intake of college students: Utilization of the health belief model. Open Journal of Preventive Medicine, 2(1). doi: 10.4236/ojpm.2012.21005.

García-González, Á., Achón, M., Alonso-Aperte, E., \& Varela-Moreiras, G. (2018). Identifying factors related to food agency: cooking habits in the spanish adult population-a cross-sectional study. Nutrients, 10(2), 217. doi: 10.3390/nu10020217

Hartmann, C., Dohle, S., \& Siegrist, M. (2013). Importance of cooking skills for balanced food 
choices. Appetite, 65. doi: 10.1016/j.appet.2013.01.016.

Isbill, J., Kandiah, J., \& Khubchandani, J. (2017). Consumers' perceptions, knowledge, and predictors of spice usage for health promotion: a pilot study of adults in midwestern united states. Journal of the Academy of Nutrition and Dietetics, 117(9), A20. doi:10.1016/j.jand.2017.06.357.

Karbownik, M. S., Paul, E., Nowicka, M., Nowicka, Z., Kowalczyk, R. P., Kowalczyk, E., \& Pietras, T. (2019). Knowledge about dietary supplements and trust in advertising them: development and validation of the questionnaires and preliminary results of the association between the constructs. PloS one, 14(6), e0218398. doi:10.1371/journal.pone.0218398

Kojuri, J., Vosoughi, A., \& Akrami, M. (2007). Effects of anethum graveolens and garlic on lipid profile in hyperlipidemic patients. Lipids in Health and Disease, 6(5). doi:10.1186/1476-511X-6-5.

Kopeć, A., Piątkowska, E., Leszczyńska, T., \& Sikora, E. (2013). Healthy properties of garlic. Current Nutrition \& Food Science, 9(1), 59-64. doi: 10.2174/157340113804810888.

Lee, S., Choi, Y., Jeong, H., Lee, J., \& Sung, J. (2017). Effect of different cooking methods on the content of vitamins and true retention in selected vegetables. Food Science and Biotechnology, 27(2), 1-10. doi:10.1007/s10068-017-0281-1

Lessa, K., Cortes, C., Frigola, A., \& Esteve, M. J. (2016). Food healthy knowledge, attitudes and practices: Survey of the general public and food handlers. International Journal of Gastronomy and Food Science, 7, 1-4. doi: 10.1016/j.ijgfs.2016.11.004.

Lobo, V., Patil, A., Phatak, A., \& Chandra, N. (2010). Free radicals, antioxidants and functional foods: Impact on human health. Pharmacognosy Reviews, 4(8), 118-126. doi:10.4103/0973$\underline{7847.70902}$

Locatelli, D., Altamirano, J., González, R., \& Camargo, A. (2015). Home-cooked garlic remains a healthy food. Journal of Functional Foods, 16, 1-8. doi: 10.1016/j.jff.2015.04.012.

Martins, C., Baraldi, L., Scagliusi, F., Villar, B., \& Monteiro, C. (2019). Cooking skills index: development and reliability assessment. Revista de Nutrição, 32. doi: 10.1590/1678-9865201932e180124.

Martins, N., Petropoulos, S., \& Ferreira, I. (2016). Chemical composition and bioactive compounds of garlic (Allium sativum L.) as affected by pre- and post-harvest conditions: A review. Food Chemistry, 211, 41-50. doi: 10.1016/j.foodchem.2016.05.029.

Mnayer, D., Fabiano-Tixier, A. S., Petitcolas, E., Hamieh, T., Nehme, N., Ferrant, C., \& Chemat, F. (2014). Chemical composition, antibacterial and antioxidant activities of six essentials oils from the Alliaceae family. Molecules (Basel, Switzerland), 19(12), 20034-20053. doi:10.3390/molecules191220034

Montaño, A., Beato, V., Mansilla, F., \& Orgaz, F. (2011). Effect of genetic characteristics and environmental factors on organosulfur compounds in garlic (allium sativum L.) grown in Andalusia, Spain. Journal of Agricultural and Food Chemistry, 59(4), 1301-7. doi:10.1021/jf104494j

Nani, M. (2016). Relationship between nutrition knowledge and food intake of college students. [Masters thesis, Kent State University]. website, https://etd.ohiolink.edu/apexprod/rws_olink/r/1501/10?clear=10\&p10 accession_num=kent1469 $\underline{155764}$

Nawaz, M., Khalid, S., \& Ahmed, S. (2016). A study to assess relationship between nutrition knowledge and food choices among young females. EC Nutrition, 6(1), 13-23. website, https://www.ecronicon.com/ecnu/pdf/ECNU-06-0000181.pdf

Petrovska, B. B., \& Cekovska, S. (2010). Extracts from the history and medical properties of 
garlic. Pharmacognosy reviews, 4(7), 106-110. doi:10.4103/0973-7847.65321

Pieniak, Z., Verbeke, W., \& Scholderer, J. (2010). Health-related beliefs and consumer knowledge as determinants of fish consumption. Journal of Human Nutrition and Dietetics: The Official Journal of the British Dietetic Association, 23(5), 480-8. doi: 10.1111/j.1365-277X.2010.01045.X.

Rahman, M. (2007). Allicin and other functional active components in garlic: health benefits and bioavailability. International Journal of Food Properties, 10(2), 245-268. doi: 10.1080/10942910601113327

Schroeter, C., House, L., \& Lorence, A. (2007). Fruit and vegetable consumption among college students in Arkansas and Florida: food culture vs. health knowledge. International Food and Agribusiness Management Review, 10(3), 63-89. doi: 10.22004/ag.econ.8165

Short, F. (2003). Domestic Cooking Skills-What Are They. Journal of the HEIA. 10. 13-22. website, https://www.mv.helsinki.fi/home/palojoki/english/GBG\%202007/francesshort.pdf

Silva, E., Moretti, C., \& Mattos, L. (2010). Functional compounds present in garlic cloves stored under refrigeration from crops in Brazil and China. Ciência Rural, 40(12). 2580-2587. website, https://periodicos.ufsm.br/cr/article/view/61474

Spronk, I., Kullen, C., Burdon, C., \& O'Connor, H. (2014). Relationship between nutrition knowledge and dietary intake. The British Journal of Nutrition, 111(10). 1-14. doi: 10.1017/S0007114514000087.

Teoh, S., Ngorsuraches, S., \& Lai, NM., Bangpan, M., \& Chaiyakunapruk, N. (2019). Factors affecting consumers' decisions on the use of nutraceuticals: a systematic review. International Journal of Food Sciences and Nutrition. 70(4) 1-22. doi:10.1080/09637486.2018.1538326.

Ternier, S. (2010). Understanding and measuring cooking skills and knowledge as factors influencing convenience food purchases and consumption. SURG Journal, 3(2) 69-76. doi: $\underline{10.21083 / \text { surg.v3i2.1122. }}$

Corresponding Author Information

Keiko Goto, PhD

California State University, Chico

Chico, CA 95929

530-898-6767

kgoto@,csuchico.edu 